\title{
Observaciones climáticas y reconstrucción instrumental. Desarrollo de productos climáticos interpolados de alta calidad

\section{Sixto Herrera ${ }^{2}$, Juan Javier Miró Pérez ${ }^{4}$ Pere Quintana Seguí ${ }^{5}$, Julián Gonzalo ${ }^{6}$, José Antonio Ruiz-Arias ${ }^{7}$, José Carlos González-Hidalgo ${ }^{8}$, José Antonio Guijarro ${ }^{3}$, José Antonio López ${ }^{1}$}

\author{
${ }^{1}$ Agencia Estatal de Meteorología (AEMET), España \\ ${ }^{2}$ Grupo de Meteorología, Dpto. de Matemática Aplicada y Ciencias Computacionales, \\ Universidad de Cantabria, España \\ ${ }^{3}$ Agencia Estatal de Meteorología (AEMET), Delegación Territorial en Baleares, España \\ ${ }^{4}$ Departament de Física de la Terra i Termodinàmica, Facultat de Física, Universitat de Valencia, España \\ ${ }^{5}$ Observatori de l'Ebre (OE), Universitat Ramon Llull - CSIC, Roquetes, España \\ ${ }^{6}$ Grupo SECF Fitoclimatología y Cambio Climático, Instituto Universitario de Investigación en Gestión \\ Forestal Sostenible - Universidad de Valladolid, Palencia, España \\ ${ }^{7}$ Grupo de Modelización de la Atmósfera y Radiación Solar, Departamento de Física, \\ y Centro de Estudios Avanzados en Energía y Medio Ambiente, Universidad de Jaén, España \\ ${ }^{8}$ Dpto. de Geografía, Universidad de Zaragoza, España
}

\begin{abstract}
Introducción
Como ha sido reflejado por el Grupo de Trabajo 1 del IPCC (IPCC, 2013) en el 5. ${ }^{\circ}$ Informe de Evaluación, las observaciones, las simulaciones con modelos y el análisis de los procesos de retroalimentación son la base para comprender y conocer el sistema climático. Con el objetivo de mejorar los datos observacionales de modo que resuelvan los requerimientos de las comunidades científica y de impactos, así como las necesidades de los responsables de las políticas de adaptación, se han desarrollado diferentes iniciativas internacionales para recolectar datos e información para los estudios climáticos (p. ej. Copernicus, previamente GMES: Global Monitoring for Environment and Security), y para proveer servicios climáticos (p. ej. Copernicus y GFCS: Global Framework for Climate Services). El principal objetivo de algunos de estos proyectos es preparar y generar información climática que englobe las necesidades de los usuarios (WMO, 2011). Dentro de estas iniciativas se han identificado un conjunto de Variables Climáticas Esenciales (ECV) (GCOS, 2010a; Bojinski et al. 2014), basadas en su relevancia para caracterizar el sistema climático y sus cambios, factibilidad y coste. También han sido generadas guías y prácticas de buen uso para obtener y dar soporte a la generación de bases de datos largas, trazables y de alta calidad de las ECV (GCOS, 2010b).
\end{abstract}

En este marco, varios proyectos han surgido en la última década para mejorar la cobertura temporal y espacial de las redes de observaciones (p. ej. proyectos EURO4M o UERRA), procesar las medidas obtenidas para aislar la señal climática (p. ej. Action Cost HOME o MeteoMet), desarrollar productos derivados (p. ej. datos interpolados en rejilla) adecuados para diferentes comunidades, e incluirlas en los procesos de asimilación de los reanálisis (p. ej. proyecto UERRA). A pesar de la mejoría de las redes de observación y del desarrollo de bases de datos de gran calidad (véase Guijarro et al., en este volumen, para más detalles), los productos interpolados de alta resolución basados en datos observacionales son cada vez más requeridos por las comunidades de impacto y de análisis climático. Varios productos han sido desarrollados dentro de las actividades de diferentes proyectos nacionales (p. ej. Portugal: Belo-Pereira et al., 2011; Rumania: Birsan and Dumitrescu, 2014; Dumitrescu and Birsan, 2015; la región alpina: Isotta et al., 2014; o Alemania: base de datos de precipitación HYRAS, Rauthe, 2013) o internacionales (p. ej. E-OBS, Haylock et al., 2008; van den Besselaar et al., 2011) aplicando diferentes métodos de interpolación a las observaciones.

Este trabajo describe los principales avances e iniciativas surgidas desde el anterior informe de CLIVAR-Spain sobre el desarrollo de productos climáticos interpolados, haciendo énfasis en aquellos estudios desarrollados para la península ibérica.

\section{Desarrollo de bases de datos en rejilla}

Como se ha descrito en la sección anterior, las bases de datos observacionales de calidad alta constan habitualmente de un número limitado de series temporales distribuidas de forma heterogénea, con representatividad local y, en la mayoría de los casos, cubriendo diferentes periodos temporales. Sin embargo, bases de datos con diferentes propiedades son necesarias para diferentes propósitos, y algún posproceso es necesario para construir productos adecuados. Bases de datos de alta resolución construidas a partir 
de bases de datos observacionales de alta calidad son demandadas por los analistas del clima y las comunidades de impacto, habiéndose desarrollado varios productos en los últimos años en respuesta a esta demanda (p. ej. E-OBS en Europa o MOPREDAS, MOTEDAS, SAFRAN o Spain02, entre otros, en España).

Debido a la alta variabilidad climática y la orografía compleja de la península ibérica, los productos internacionales disponibles (p. ej. E-OBS, WATCH o WFDEI) no son capaces de reproducir adecuadamente los diferentes regímenes climáticos de la península ibérica (Herrera et al., 2012, 2015; Bedia et al., 2013). Varias bases de datos regionales y nacionales han sido desarrolladas en la última década usando diferentes aproximaciones de acuerdo a las necesidades de las comunidades científica y de impacto, dando lugar a un amplio rango de productos que introduciremos en esta sección.

En lo que respecta a bases de datos de muy alta resolución, Niyerola et al. (2007) desarrollaron una base de datos con una resolución espacial de 200 m para la península ibérica de climatologías mensuales y anual de precipitación, radiación y temperaturas, que ha sido muy utilizada en el estudio de modelos de nicho ecológico y de distribución de especies. Por su parte, Gonzalo et al. (2010) generaron una base de datos similar para el desarrollo de un diagnóstico fitoclimático de la península ibérica, pero usando un método diferente de interpolación (cokriging) y otras variables explicativas, y alcanzando una resolución inferior $(1 \mathrm{~km})$.

A escala regional, Vicente-Serrano et al. (2003, 2007 y 2010) han desarrollado mapas climáticos mensuales de precipitación, temperaturas y niebla en el Valle del Ebro y Aragón con una resolución de $1 \mathrm{~km}$ considerando varios métodos de interpolación; Garzón-Machado et al. (2014) construyeron un mapa de vegetación potencial natural del climatófilo en la isla de La Palma (Canarias) a una resolución espacial de 25 m; Ruiz-Arias et al. (2011, 2015 y 2016) desarrollaron dos bases de datos interpoladas de radiación solar para Andalucía $(1 \mathrm{~km})$ y la España peninsular y las islas Baleares $(10 \mathrm{~km})$, usando esta última para validar la radiación solar reflejada por el modelo regional del clima WRF. Sin embargo, uno de los objetivos de la mayoría de estos estudios era la intercomparación de los métodos de interpolación y, en consecuencia, la mayoría de estas bases de datos no está disponible o lo está previo requerimiento a los autores.

A pesar de la alta resolución espacial de las bases de datos descritas, en multitud de ocasiones se requiere de series temporales de dato mensual (p. ej. para realizar análisis de tendencias), diario o incluso intradiario. En esta línea, González-Hidalgo et al. (2011 y 2015) desarrollaron bases de datos mensuales de precipitación (MOPREDAS) y temperaturas (MOTEDAS) cubriendo los periodos 1945-2005 y 1951-2010, respectivamente, para realizar análisis de tendencias, validación de modelos y regionalización estadística en la España peninsular. Ambas bases de datos tienen una resolución espacial de $0,1^{\circ}$ y han sido construidas considerando una red muy densa de observaciones, con 2670 estaciones para precipitación y 1358 para temperatura, pertenecientes a la red de la Agencia Estatal de Meteorología (AEMET).
A escala diaria, Herrera et al. (2011, 2012 y 2016) desarrollaron un conjunto de bases de datos interpolados de alta resolución de precipitación y temperaturas (Spain02) para la España peninsular y las islas Baleares. Para ello consideraron 2756 estaciones de precipitación y 250 estaciones de temperatura incluidas en la red secundaria de AEMET cumpliendo diferentes criterios de calidad. En particular, la versión v2 (rejilla regular de $0,2^{\circ}$ de resolución) es una base de datos interpolados con representatividad local, apropiada para análisis locales y de eventos extremos (Herrera et al., 2012), mientras que la version v4 incluye tres resoluciones diferentes coincidiendo con las rejillas definidas en el marco de Euro-CORDEX $\left(0,11^{\circ}, 0,22^{\circ}\right.$ y $0,44^{\circ}$ en coordenadas rotadas). Esta versión contiene valores representativos del promedio areal (AA, promediados de una rejilla auxiliar de $0,01^{\circ}$ de resolución espacial) en los productos AA-2D, AA-3D y AA-OK, siguiendo la notación definida en Herrera et al. (2016), siendo un producto apropiado para la validación de modelos regionales del clima (RCM). En esta versión también se incluyen productos con representatividad local (OK, Kriging Ordinario) equivalentes a los desarrollados en la v2. Como complemento a la v2, se desarrolló una versión v3 considerando un subconjunto de estaciones con series largas de precipitación con muy pocas lagunas (al menos 40 años con a lo sumo el $10 \%$ de datos perdidos por año), siendo adecuada para el análisis de tendencias en el periodo considerado. Las diferentes versiones de Spain02 se distribuyen públicamente para investigación a través del portal de servicios climáticos de AEMET. La versión v2 fue la base de datos de referencia en el desarrollo de los escenarios regionalizados de cambio climático en el marco del programa nacional PNACC-2012. Por otra parte, la version v4 fue una de las bases de datos interpoladas nacionales consideradas en el marco de la iniciativa COST Action VALUE (http://www.value-cost.eu/), usadas para validar los modelos regionales del clima de Euro-CORDEX, la rama Europea de la iniciativa CORDEX (http://cordex.org), y analizar la incertidumbre observacional asociada a la base de datos de referencia considerada en la evaluación del modelo.

Como complemento a las bases de datos interpoladas de España, Belo-Pereira et al. (2011) desarrollaron una base de datos de precipitación para Portugal (PT02) usando 400 estaciones de calidad y considerando la misma técnica de interpolación y rejilla usada en la versión v2 de Spain02. Por lo tanto, ambas bases de datos pueden considerarse equivalentes en el periodo común, 1951-2003, siendo combinadas en estudios recientes para obtener una base de datos de la península ibérica, IB02, de precipitación diaria (Ramos et al., 2016; Sousa et al., 2016).

Recientemente, los trabajos realizados en Francia para desarrollar la base de datos de análisis SAFRAN (Durand et al., 1993; 1999) han sido aplicados para la España peninsular y las islas Baleares (Quintana-Seguí et al., 2016; 2017), obteniendo una base de datos interpolada de alta resolución espacial $(5 \mathrm{~km})$ y temporal (horaria) basada en precipitación diaria y temperatura, viento, humedad relativa y nubosidad 6-horarias. SAFRAN también incluye la radiación recibida modelada, tanto visible como infrarroja. De este modo, SAFRAN incluye todas las variables necesarias para forzar un modelo de superficie (LSM, por sus 
siglas en inglés, Land-Surface Model) o cualquier otro modelo hidrológico basado en las citadas variables. La cobertura temporal abarca el periodo 1979-2014, y está disponible para investigación a través de la base de datos Mistrals-HyMex (Quintana-Seguí, 2015).

A escala regional, varias bases de datos diarias de alta resolución espacial $(\sim 1 \mathrm{~km})$ han sido generadas en los últimos años dentro de proyectos regionales o necesidades y/o análisis específicos de diferentes grupos de investigación. Militino et al. (2015) definieron y validaron un método de interpolación espacio-temporal para obtener una rejilla de precipitación de $1 \mathrm{~km}$ de resolución cubriendo la región de Navarra. Dentro del proyecto regional «Escenarios Regionales Probabilísticos de Cambio Climático en Cantabria: Termopluviometría», Gutiérrez et al. (2010) desarrollaron un producto similar para Cantabria, incluyendo precipitación y temperatura, el cual fue utilizado en el marco del proyecto para desarrollar los escenarios regionales de cambio climático para la región aplicando técnicas de regionalización estadística. Por su parte, Miró Pérez et al. (2015) combinaron métodos de regionalización estadística e interpolación espacial para obtener una base de datos diaria de muy alta resolución de temperaturas máxima y mínima para la región de Valencia considerando alrededor de 300 estaciones de diferentes instituciones (AEMET, CEAM, SIAR e IIG) cubriendo el periodo 1948-2011. Esta base de datos es referida como SDSITVC en Miró et al. (2016), donde es propuesta como una herramienta para estimar el cambio bioclimático ocurrido en las áreas de montaña de la región.

\section{Resumen y conclusiones}

En los últimos años se ha realizado un gran esfuerzo para mejorar la calidad de las herramientas y productos usados para analizar el sistema climático desde diferentes puntos de vista. En esta línea, varias iniciativas y proyectos se han propuesto y llevado a cabo con el objetivo de rescatar y digitalizar datos observacionales existentes, con especial atención a regiones con cobertura espacial y/o temporal insuficiente, y desarrollar herramientas y métodos adecuados para elaborar bases de datos de alta calidad para el análisis del clima.

En este marco, la comunidad climática española está involucrada en la mayoría de los proyectos e iniciativas actuales relacionadas con el desarrollo de herramientas y productos (p. ej., librería Climatol para el análisis y homogenización de datos, o las bases de datos interpoladas MOPREDAS, SAFRAN o Spain02), los cuales son utilizados por la comunidad internacional en gran variedad de estudios, y con la extensión de los métodos de asimilación de datos a nuevos modelos, variables y procesos.

En particular, como se ha reflejado en este artículo, varios productos en rejilla regionales y nacionales han sido desarrollados en los últimos años abarcando un gran número de aplicaciones, resoluciones, variables y periodos temporales. Sin embargo, el principal inconveniente a señalar es que la mayoría de estas bases de datos raramente se ponen a disposición de la comunidad científica, dando lugar a análisis redundantes/replicados en muchos casos, y limitando el impacto y los análisis realizados sobre dichas bases de datos. Por otro lado, no existe actualmente un análisis de intercomparación adecuado entre las diferentes bases de datos desarrolladas. Finalmente, los cambios en la política de distribución de datos de la Agencia Estatal de Meteorología, junto con los avances y desarrollo en las herramientas de análisis de datos permite la publicación futura de nuevos y actualizados productos interpolados para el análisis climático.

\section{Referencias}

Bedia, J., Herrera, S., Gutiérrez, J. M., 2013: Dangers of using global bioclimatic datasets for ecological niche modeling. Limitations for future climate projections, Global and Planetary Change, 107, 1-12, doi: 10.1016/ j.gloplacha.2013.04.005.

Belo-Pereira, M., Dutra, E. and Viterbo, P., 2011: Evaluation of global precipitation data sets over the Iberian Peninsula, J. Geophys. Res., 116, D20101, doi:10.1029/ 2010JD015481.

Birsan, M. V., Dumitrescu, A., 2014: ROCADA: Romanian daily gridded climatic dataset (1961-2013) V1.0. Administratia Nationala de Meteorologie, Bucuresti, Romania, doi:10.1594/PANGAEA.833627.

Bojinski, S., Verstraete, M., Peterson, T. C., Richter, C., Simmons, A., Zemp, M., 2014: The concept of essential climate variables in support of climate research, applications, and policy. Bull. Amer. Meteor. Soc., 95, 1431-1443, doi:10.1175/BAMS-D-13-00047.1.

Dumitrescu, A., Birsan, M. V., 2015: ROCADA: a gridded daily climatic dataset over Romania (1961-2013) for nine meteorological variables. Natural Hazards, 78 (2), 1045-1063, doi:10.1007/s11069-015-1757-z.

Durand, Y., Brun, E., Merindol, L., Guyomarc'h, G., Lesaffre, B., Martin, E., 1993: A meteorological estimation of relevant parameters for snow models, Ann. Glaciol., 18, 65-71.

Durand, Y., Giraud, G., Brun, E., Merindol, L., Martin, E., 1999: A computer-based system simulating snowpack structures as a tool for regional avalanche forecasting, J. Glaciol., 45 (151), 469-484.

Garzón-Machado, V., Otto, R., del Arco Aguilar, M. J., 2014: Bioclimatic and vegetation mapping of a topographically complex oceanic island applying different interpolation techniques, Int. J. Biometeorol., 58, 887-899, doi: DOI 10.1007/s00484-013-0670-y.

GCOS, 2010a: Implementation plan for the global observing system for climate in support of the UNFCCC (2010 update). GCOS Rep. 138, 186 pp. Disponible en línea en: www.wmo.int/pages/prog/gcos/Publications/ gcos-138.pdf.

GCOS, 2010b: Guideline for the generation of datasets and products meeting GCOS requirements. GCOS Rep. 143, 12 pp. Disponible en línea en: www.wmo.int/pages/prog/ gcos/Publications/gcos-143.pdf. 
Gonzalo, J., 2010: Diagnosis Fitoclimática de la España Peninsular. Hacia un modelo de clasificación funcional de la vegetación y de los ecosistemas peninsulares españoles. Organismo Autónomo de Parques Nacionales-Botánica General, ISBN: 978-84-8014-787-3.

González-Hidalgo, J. C., Brunetti, M., de Luis, M., 2011: A new tool for monthly precipitation analysis in Spain: MOPREDAS database (monthly precipitation trends December 1945-November 2005). Int. J. Climatol., 31, 715-731. doi:10.1002/joc. 2115.

González-Hidalgo, J. C., Peña-Angulo, D., Brunetti, M., Cortesi, N., 2015: MOTEDAS: a new monthly temperature database for mainland Spain and the trend in temperature (1951-2010). Int. J. Climatol., 35, 4444-4463. doi:10.1002/ joc.4298.

Guijarro, J. A., Herrera, S., 2017: Climatic observations and instrumental reconstructions I: development of highquality climatic time series.

Gutiérrez, J. M., Herrera, S., San-Martín, D., Sordo, C., Rodríguez, J. J., Frochoso, M., Ancell, R., Fernández, J., Cofiño, A. S., Pons, M. R., Rodríguez, M. A., 2010: Escenarios Regionales Probabilísticos de cambio climático en Cantabria: Termopluviometría, Gobierno de CantabriaConsejería de Medio Ambiente y Universidad de Cantabria, Santander, España.

Haylock, M. R., Hofstra, N., Klein Tank, A. M. G., Klok, E. J., Jones, P. D., New, M., 2008: A European daily highresolution gridded dataset of surface temperature and precipitation. J. Geophys. Res. (Atmospheres), 113, D20119, doi:10.1029/2008JD10201.

Herrera, S., 2011: Desarrollo, validación y aplicaciones de Spain02: Una rejilla de alta resolución de observaciones interpoladas para precipitación y temperatura en España. Tesis doctoral. Universidad de Cantabria, Cantabria, Spain. http://www.meteo.unican.es/tesis/herrera.

Herrera, S., Gutiérrez,J. M., Ancell, R., Pons, M. R.,Frías, M. D., Fernández, J., 2012: Development and Analysis of a 50 year high-resolution daily gridded precipitation dataset over Spain (Spain02), Int. J. Climatol., 32, 74-85, doi: 10.1002/joc.2256.

Herrera, S., Fernández, J., Gutiérrez, J. M., 2016: Update of the Spain02 Gridded Observational Dataset for EuroCORDEX evaluation: Assessing the Effect of the Interpolation Methodology, Int. J. Climatol., 36, 900-908, doi: 10.1002/joc. 4391 .

IPCC, 2013: Climate Change 2013: The Physical Science Basis. Contribution of Working Group I to the Fifth Assessment Report of the Intergovernmental Panel on Climate Change [Stocker, T. F., D. Qin, G.-K. Plattner, M. Tignor, S. K. Allen, J. Boschung, A. Nauels, Y. Xia, V. Bex and P. M. Midgley (eds.)]. Cambridge University Press, Cambridge, United Kingdom and New York, NY, USA, 1535 pp.

Isotta, F. A., Frei, C., Weilguni, V., Perèec Tadiæ, M., Lassègues, P., Rudolf, B., Pavan, V., Cacciamani, C.,
Antolini, G., Ratto, S. M., Munari, M., Micheletti, S., Bonati, V., Lussana, C., Ronchi, C., Panettieri, E., Marigo, G., Vertaènik, G., 2014: The climate of daily precipitation in the Alps: development and analysis of a high-resolution grid dataset from pan-Alpine rain-gauge data. Int. J. Climatol., 34, 1657-1675. doi:10.1002/joc.3794.

Militino, A. F., Ugarte, M. D., Goicoa, T., Genton, M., 2015: Interpolation of daily rainfall using spatiotemporal models and clustering, International Journal of Climatology, 35, 1453-1464, doi:10.1002/joc.4068.

Miró Pérez, J. J., Estrela Navarro, M. J., Olcina-Cantos, J., 2015: Statistical downscaling and attribution of air temperature change patterns in the Valencia region (1948-2011), Atmospheric Research, 156, 189-212.

Miró Pérez, J. J., Estrela Navarro, M. J., Caselles, V., Olcina-Cantos, J., 2016: Fine-scale estimations of bioclimatic change in the Valencia region, Spain. Atmospheric Research, 180, 150-164. doi:10.1016/j.atmosres.2016.05.020.

Ninyerola, M., Pons, X., Roure, J. M., 2007: Monthly precipitation mapping of the Iberian Peninsula using spatial interpolation tools implemented in a Geographic Information System. Theoretical and Applied Climatology, 89, 195-209.

Pérez, F. F., Boscolo, R., 2010: Climate in Spain: past, present and future. Regional climate change assessment report. Spanish CLIVAR Committee / Thematic network. http://www.clivar.org/sites/default/files/documents/ CLIVAR_Spain_2010_0.pdf.

Quintana-Seguí, P., 2015: SAFRAN analysis over Spain, doi:10.14768/MISTRALS-HYMEX.1388.

Quintana-Seguí, P., Peral, C., Turco, M., Llasat, M. C. and Martin, E., 2016: Meteorological Analysis Systems in North-East Spain: Validation of SAFRAN and SPAN, Journal of Environmental Informatics, 27 (2), 116-130, doi:10.3808/jei.201600335.

Quintana-Seguí, P., Turco, M., Herrera, S., Miguez-Macho, G., 2017: Validation of a new SAFRAN-based gridded precipitation product for Spain and comparison to Spain02 and ERA-Interim. Hydrology and Earth System Sciences, 21, 2187-2201, doi: 10.5194/hess-21-2187-2017.

Ramos, A. M., Trigo, R. M. and Liberato, M. L. R., 2017, Ranking of multi-day extreme precipitation events over the Iberian Peninsula. Int. J. Climatol., 37, 607-620. doi:10.1002/ joc. 4726 .

Rauthe, M., Steiner, H., Riediger, U., Mazurkiewicz, A., Gratzki, A., 2013: A Central European precipitation climatology Part I: Generation and validation of a high-resolution gridded daily data set (HYRAS), Meteorologische Zeitschrift, 22 (3), 235-256, doi: 10.1127/0941-2948/2013/0436.

Ruiz-Arias, J. A., Pozo-Vázquez, D., Santos-Alamillos, F. J., Lara-Fanego, V., Tovar-Pescador, J., 2011: A topographic geostatistical approach for mapping monthly mean 
values of daily global solar radiation: A case study in southern Spain, Agricultural and Forest Meteorology, 151, 1812-1822.

Ruiz-Arias, J. A., Quesada-Ruiz, S., Fernández, E. F., Gueymard, A., 2015: Optimal combination of gridded and ground-observed solar radiation data for regional solar resource assessment, Solar Energy, 112, 411-424.

Ruiz-Arias, J. A., Arbizu-Barrena, C., Santos-Alamillos, F. J., Tovar-Pescador, J., Pozo-Vázquez, D., 2016: Assessing the surface solar radiation budget in the WRF Model: a spatiotemporal analysis of the bias and its causes, Monthly Weather Review, 144, 703-711.

Sousa, P. M., Barriopedro, D., Trigo, R. M., Ramos, A. M., Nieto, R., Gimeno, L., Turkman, K. F., Liberato, M. L. R., Impact of Euro-Atlantic blocking patterns in Iberia precipitation using a novel high resolution dataset, Climate Dynamics, 2016, 46, 7-8, 2573.

Van den Besselaar, E. J. M., Haylock, M. R., van der Schrier, G., Klein Tank, A. M. G., 2011: A European Daily High-resolution Observational Gridded Data set of Sea
Level Pressure. J. Geophys. Res., 116, D11110, doi:10.1029/2010JD015468.

Vicente-Serrano, S. M., Saz-Sánchez, M. A., Cuadrat, J. M., 2003: Comparative analysis of interpolation methods in the middle Ebro Valley (Spain): application to annual precipitation and temperature, Climate Research, 24 (2), 161-180, doi: 10.3354/cr024161.

Vicente-Serrano, S. M., Lanjeri, S., López-Moreno, J. I., 2007: Comparison of different procedures to map reference evapotranspiration using geographical information systems and regression-based techniques. Int. J. Climatol., 27, 1103-1118. doi:10.1002/joc.1460.

Vicente-Serrano, S. M., Beguería, S., López-Moreno, J. I., García-Vera, M. A., Štepánek, P., 2010: A complete daily precipitation database for northeast Spain: reconstruction, quality control, and homogeneity. Int. J. Climatol., 30, 1146-1163. doi:10.1002/joc.1850.

WMO, 2011: World Meteorological Organization, 2011: Guide to Climatological Practices, WMO-No. 100, ISBN 978-92-63-10100-6, Geneva. 